\title{
Retos de la seguridad en caso de incendio de levantes en edificios de vivienda existentes
}

\section{Fire safety challenges of floor additions in existing housing blocks}

Juan Bautista Echeverría $^{(*)}$, Nieves Rubio ${ }^{(*)}$, Itziar Rodríguez ${ }^{(*)}$, Jimmy Jönsson ${ }^{(*)}$, Gabriele Vigne ${ }^{(* *)}$, Javier Sánchez ${ }^{(* *)}$

\section{RESUMEN}

La adición de pisos(levantes) en edificios existentes de viviendas ha sido una práctica habitual en Europa desde mediados del siglo XX. Los desarrollos urbanos en los ensanches, ocupados por bloques de viviendas en la segunda mitad del siglo XIX y en la primera del XX, encuentran en los levantes una oportunidad para cubrir los costes de renovación y para limitar la dispersión urbana. Sin embargo, las obras para llevarlos a cabo enfrentan a promotores, diseñadores y reguladores a muchos retos, constituyendo la seguridad de incendio (SCI) uno de los más confusos. Los Códigos prescriptivos de SCI están redactados para edificios nuevos y su uso en edificios existentes puede tener resultados impredecibles, llegando incluso a imposibilitar determinadas intervenciones. Este artículo analiza la problemática que la aplicación de la regulación prescriptiva española de SCI genera en los levantes, proponiendo soluciones basadas en prestaciones que no disminuyan el nivel de seguridad requerido.

Palabras clave: Levantes, seguridad en caso de incendio, análisis prestacional, seguridad equivalente.

\section{ABSTRACT}

Floor additions in existing housing blocks have been an extended practice in Europe since the middle of the $2 O^{\text {th }}$ century. The urban developments in the city expansions, occupied by housing blocks in the second half of the $19^{\text {th }}$ century and the first half of the $2 O^{\text {th }}$, find in the additions an opportunity, both to cover the renovation costs and to limit urban sprawl. However, the works to carry them up, face developers, designers and code officials with many challenges, being fire safety (FS) one of the most confusing. Prescriptive Fire Safety Codes are intended for new buildings, and their use in existing ones may have unpredictable results, even making hopeless some interventions. This paper analyses the problematic that the application of the Spanish FS prescriptive regulation generates in floors additions, proposing performance-based solutions that do not diminish the required safety level.

Keywords: Floor additions, fire safety, performance-based analysis, equivalent safety.

${ }^{*}$ ) Colegio Oficial de Arquitectos Vasco Navarro, San Sebastián, España

$\left.{ }^{* *}\right)$ JVVA Fire \& risk, Madrid, España

Persona de contacto/Corresponding author: juanecheve@coavn.org (J. B. Echeverría)

ORCID: http://orcid.org/oooo-00o2-4438-6449 (J. B. Echeverría); http://orcid.org/oooo-00o2-6175-8224 (N. Rubio);

http://orcid.org/oooo-0002-6327-0046 (I. Rodríguez); http://orcid.org/oooo-0003-2227-6879 (J. Jönsson);

http://orcid.org/oooo-0002-0887-0989 (G. Vigne); https://orcid.org/oooo-0001-9550-0363 (J. Sánchez)

Cómo citar este artículo/Citation: Echeverría, Juan Bautista; Rubio, Nieves; Rodríguez, Itziar; Jönsson, Jimmy; Vigne, Gabriele; Sánchez, Javier (2020). Retos de la seguridad en caso de incendio de levantes en edificios de vivienda existentes. Informes de la Construcción, $72(559)$ : e351. https://doi.org/10.3989/ic.73265

Copyright: (C) 2020 CSIC. Este es un artículo de acceso abierto distribuido bajo los términos de la licencia de uso y distribución Creative Commons Reconocimiento 4.0 Internacional (CC BY 4.0). 


\section{INTRODUCCIÓN}

Los centros de las ciudades constituyen hoy importantes polos de interés por su localización, su dinamismo y su oferta cultural y turística. En muchas ciudades europeas, son el fruto de ensanches que, partiendo de pequeños núcleos más antiguos, se desarrollaron a lo largo de los siglos XIX y XX con un uso fundamentalmente residencial. La presión urbanística sobre ellos fue colmatándolos, introduciendo además usos administrativos, comerciales, culturales y de ocio. Sin embargo, el interés por habitar esos centros se mantiene para muchos usuarios.

La construcción de levantes de unas pocas plantas sobre los bloques residenciales existentes (en muchos casos de poca altura, teniendo en cuenta que muchos se diseñaron de forma previa o en los primeros tiempos del ascensor) constituye, desde hace años, una forma de respuesta a esa demanda. En el caso concreto del País Vasco, ciudades como Donostia-San Sebastián, han intensificado esa práctica desde la mitad del siglo XX por su privilegiada situación geográfica en la costa que incrementaba el precio del suelo.

Los levantes sobre lo ya construido y consolidado tienen, además, dos importantes ventajas que se alinean con cualquier estrategia de sostenibilidad: colaboran, en primer lugar, a densificar la ciudad sin consumir suelo nuevo, facilitando, en segundo lugar, la regeneración urbana, al permitir que los edificios se renueven y mejoren sus prestaciones (1). Es importante hacer notar que la evolución de la proporción de los hogares por tamaño señala una tendencia muy clara a una disminución en el tamaño medio de los hogares (2). Concretamente, en 2033 los hogares en los que vivirán una o dos personas supondrán un 60,4\% del total, por lo que aumentar el número de viviendas mediante operaciones de levante no conllevará necesariamente un aumento de usuarios en los edificios en los que se intervenga.

\section{CARACTERÍSTICAS GENERALES DE LOS EDIFICIOS DE VIVIENDAS SUSCEPTIBLES DE SER AMPLIADOS MEDIANTE LEVANTES}

Los edificios susceptibles de ser objeto de levantes responden a una tipología de manzana cerrada en la que se insertan bloques entre medianerías. El perfil habitual suele ser de planta baja más cuatro o cinco plantas de pisos, incorporando también, con frecuencia, una planta de sótano y una planta bajo la cubierta (Figura 1).

La planta baja, además de los accesos a las viviendas, suele tener usos comerciales o de pública concurrencia, que a su vez suelen comunicar con el sótano, estando el resto del edificio destinado a uso de vivienda. Es común encontrar dos viviendas por planta con patios interiores para iluminación y ventilación, que a veces son compartidos con los bloques adyacentes. La escalera suele dar también a algún patio.

La estructura portante es con frecuencia mixta, con pilares interiores, vigas y viguetas de madera, que se completa con muros de piedra en fachadas y medianeras. Las instalaciones son básicas y se corresponden con las que eran habituales en su momento de construcción: abastecimiento y evacuación de aguas y sencillas instalaciones eléctricas.

Si analizamos el estado de conservación de estos edificios, podemos reseñar que en muchos casos son edificios que han recibido un mantenimiento bastante irregular, encontrándose los
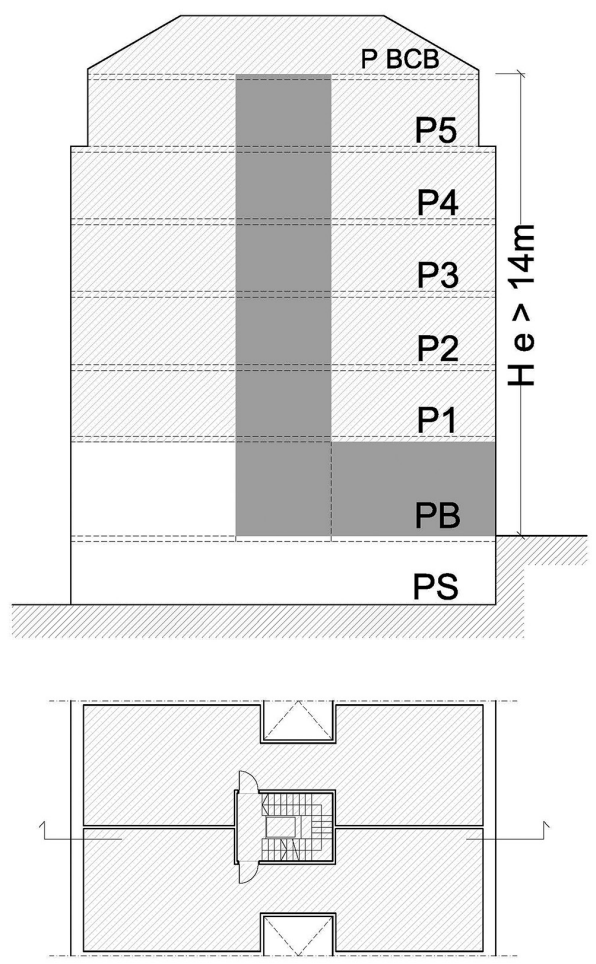

Figura 1. Sección y Planta tipo de edificio susceptible de ser objeto de levante.

interiores de las viviendas en bastante buen estado frente a los elementos comunes, que han ido envejeciendo sin muchas actuaciones. Las mejoras de cierta envergadura se han ido acometiendo cuando se observaban patologías graves. Por esta razón, encontramos edificios en los que la instalación de un ascensor se puede considerar la intervención más remarcable. Normalmente, son edificios que carecen de instalaciones de protección contra incendios y de alumbrado de emergencia y donde quizá la instalación eléctrica se ha renovado, aun dejando los contadores en rellanos de escalera o en otras zonas comunes.

Recientemente este panorama tiene tendencia a cambiar, desde la entrada en vigor de las Inspecciones Técnicas de Edificios que están suponiendo una puesta a punto de éstos y una planificación de su futuro mantenimiento.

En los primeros levantes que se fueron realizando, las plantas añadidas se asentaban sobre la estructura existente de madera. Las propuestas no contemplaban la intervención en el edificio existente, creando, en algunos casos, una sobrecarga para la que el edificio no estaba dimensionado. Por otra parte, la carencia de normativa en cuanto a protección contra incendios permitía la obtención de licencia para la ejecución de estas intervenciones.

\section{RIESGO DE INCENDIO EN LOS EDIFICIOS DE VIVIENDAS SUSCEPTIBLES DE SER AMPLIADOS MEDIANTE LEVANTES}

Aunque el Real Decreto 1053/1985 (3) establecía la obligación en España de trasladar los datos a un Parte Unificado de Actuación, la realidad es que no se han publicado estadísticas oficiales de incendios desde 1994.

En los últimos años, los informes elaborados por la Fundación MAPFRE, en colaboración con la APTB (4), han reco- 
gido datos de víctimas mortales de incendio, analizando aspectos como la edad, el género, los meses, la hora del día y la causa del incendio. Un reciente estudio de la Universidad de Navarra (5), que se ha basado en la metodología seguida por la NFPA en los Estados Unidos, ha analizado todos los incendios con víctimas en España en 2016 a partir de noticias publicadas en medios de comunicación. Esto incluye los incendios con víctimas mortales, como en el caso de MAPFRE, pero también víctimas no mortales. Además, analiza otras variables de enorme interés (nivel de ingresos económicos de la zona, año de construcción del edificio, etc.). Entre las conclusiones más importantes del estudio se encuentran las causas más importantes de los incendios con víctimas en viviendas, reflejadas en la Tabla 1.

Este mismo estudio, que ha sido realizado fundamentalmente para evidenciar la vulnerabilidad de las personas de edad avanzada en viviendas en España, concluye que, en 2016, el grupo de población de 65 años o más, pese a constituir un 18,9\% de la población general, ha representado el 60,6\% de las víctimas mortales de incendio. También evidencia que existe un riesgo asociado a la antigüedad del edificio, teniendo los edificios construidos hace más de 65 años un riesgo relativo que duplica el de los edificios construidos en los últimos 5 años.

$\mathrm{Si}$, partiendo de esta base, caracterizamos el riego de incendio de muchos de los edificios de viviendas en los que se podrían ejecutar operaciones de levantes, podemos afirmar que se trata de edificios antiguos. Carecen en su mayoría de aislamiento térmico, por lo que sus usuarios se ven obligados en muchas ocasiones a utilizar sistemas de calefacción poco recomendables. Tienen con frecuencia un equipamiento eléctrico anticuado, especialmente en los elementos comunes, no disponiendo de sistemas de protección ni de cuartos de contadores cerrados. Además, en muchas ocasiones están habitados por personas de edad avanzada.

\section{REGULACIÓN DE LA S.C.I. EN LOS EDIFICIOS SUSCEPTIBLES DE SER AMPLIADOS MEDIANTE LEVANTES}

Con la entrada en vigor de las diferentes regulaciones de SCI aprobadas en España en la última parte del Siglo XX y en la primera del XXI, la posibilidad de intervenir en edificios existentes mediante una ampliación por levante se ha ido complicando progresivamente.

Aunque las anteriores Normas Básicas, CPI 81 (6), CPI 91 (7) y CPI 96 (8), estuvieron abiertas, en cierta medida, a cláusulas de equivalencia, el enfoque prescriptivo primó de forma abrumadora en su aplicación. El Código Técnico de la Edificación (CTE), aprobado por Real Decreto 314/2006 (9), en vigor, se alinea de forma mucho más decidida con el enfoque basado en prestaciones $(10,11)$, que es propugnado por Organizaciones Internacionales, como el Consejo Internacional de la Edificación o el Comité Interjurisdiccional de Colaboración Reglamentaria.
La regulación prescriptiva de la SCI ha tenido un indudable efecto positivo en la reducción del riesgo de incendio experimentada en España desde la implantación de la primera Norma Básica. Al ofrecer soluciones aceptables que, por propia definición, satisfacen las exigencias, son fácilmente interpretables y ejecutables por los agentes que intervienen en el proceso de la edificación. Sin embargo, la complejidad de algunos edificios y, especialmente, la intervención en edificios existentes, imposibilitan en algunos casos y desaconsejan en otros (12), su aplicación. Este es el caso de los levantes, en los que la aplicación estricta de la solución prescriptiva, exige una morfología muy concreta a las vías de evacuación, pero no parece suficientemente estricta con la capacidad portante (en situación de incendio) de la estructura en la parte existente.

Como se verá más adelante, uno de los mayores problemas que se plantea en los proyectos de levantes es la relación entre la parte construida con anterioridad a la intervención y la parte ampliada tras la misma. Al tratarse de edificios en los que la propiedad es múltiple, resulta muy difícil poner de acuerdo a los propietarios existentes para realizar modificaciones sustanciales en sus viviendas originales, máxime si esto implica una pérdida de la superficie.

Algunas administraciones autonómicas y/o locales, han tratado de dar respuesta a este problema con la publicación de documentos que puedan servir de guía para abordar la problemática que se aborda en este artículo. Es el caso del documento Remuntes en edificis d'habitatges amb la creació de nous habitatges (13) redactado en Cataluña por la Taula d'interpretació de la Normativa de Seguretat Contra Incendis (TINSCI). La normativa prescriptiva se ha venido interpretando, además, con matices diferentes desde las distintas administraciones, contribuyendo a una mayor confusión.

No se puede dejar de mencionar aquí la disposición final undécima de la Ley 8/2013, de 26 de junio, de rehabilitación, regeneración y renovación urbanas (14), que modificó el CTE en el sentido de que cuando su aplicación no sea urbanística, técnica o económicamente viable o, en su caso, sea incompatible con la naturaleza de la intervención o con el grado de protección del edificio se debe procurar el mayor grado posible de adecuación efectiva.

El presente trabajo, trata de abordar la problemática de la Seguridad Contra Incendios de los levantes desde un punto de vista prestacional, tal y como se contempla en el Artículo 5.3 del CTE. Para ello, y con el fin de garantizar que las prestaciones de las soluciones alternativas que se planteen sean, al menos, equivalentes a las que se obtendrían con la aplicación de los Documentos Básicos, se hace imprescindible la utilización de métodos cuantitativos. De esta manera se puede comparar el nivel de riesgo de cada solución adoptada y, por tanto, de las prestaciones obtenidas en caso de incendio.

Tabla 1. Porcentaje de incendios con víctimas, mortales y no, en viviendas en España (2016).

\begin{tabular}{|c|c|c|c|}
\hline Causa del incendio & Incendios & Muertos & Heridos \\
\hline Equipos de calefacción, especialmente los de llama abierta y braseros eléctricos & 14,9 & 31,8 & 19,9 \\
\hline Equipos eléctricos en mal estado & 29,4 & 22,7 & 33,3 \\
\hline Cigarrillos & 3,4 & 18,2 & 3,3 \\
\hline Chimeneas & 12,4 & 11,4 & 3,4 \\
\hline
\end{tabular}


Los análisis a realizar son:

- Análisis prescriptivo por la aplicación del DB SI

- Análisis prestacional cuantitativo utilizando el documento reconocido de análisis de riesgo MEREDICTE (15).

- Análisis prestacional de riesgos cuantitativo, utilizando herramientas de simulación de incendio y evacuación de ocupantes.

\section{ANÁLISIS PRESCRIPTIVO POR APLICACIÓN DEL DB SI}

Como ya se ha indicado, los edificios susceptibles de la realización de levantes, tienen un perfil que se corresponde aproximadamente con la sección de la Figura 1. (sótano + planta baja +4 plantas + planta bajocubierta). Al tratarse de edificios construidos hace prácticamente un siglo, ya han sufrido intervenciones de diversa índole. En algunos casos incluso se han realizado levantes de una planta, pasando a un perfil de $\mathrm{PB}+5+$ bajocubierta, $\mathrm{y}$, en prácticamente todos los casos, se ha instalado un ascensor en el ojo de la escalera.

Aplicando la normativa de manera prescriptiva, debemos cumplir el DBSI en todos sus términos. De acuerdo con los Criterios Generales de aplicación del documento, en las obras de reforma en las que se mantenga el uso, el DB debe aplicarse a los elementos del edificio modificados por la reforma. En nuestro caso, afectaría a la zona ampliada mediante levante, pero también a los elementos de evacuación del conjunto, al verse alterada la ocupación o su distribución con respecto a los elementos de evacuación. La Figura 2 representa, en sección, las zonas del edificio ampliado en las que debe aplicarse el DB-SI.

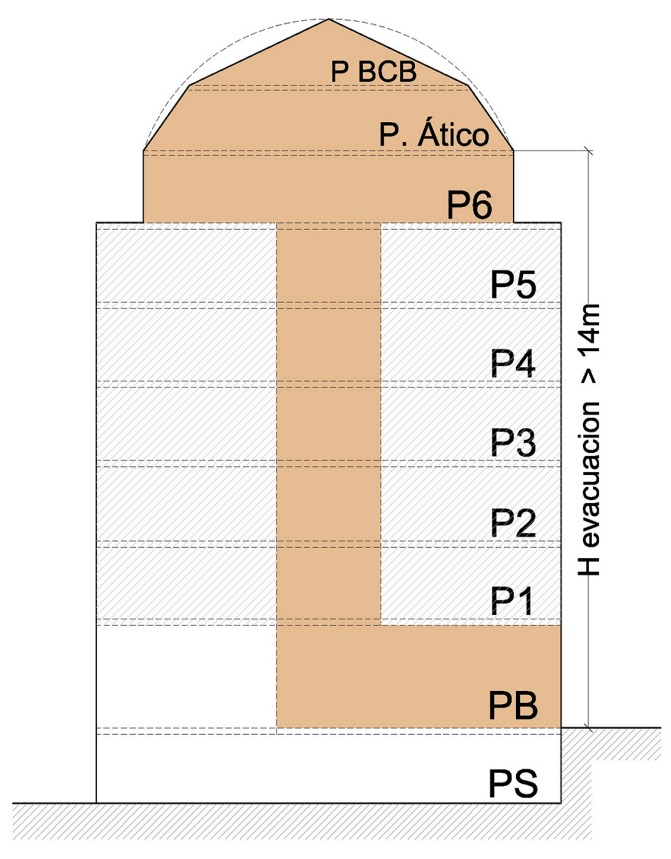

Figura 2. Sección tipo de edificio de viviendas con levante.

La Tabla 2 recoge las exigencias del DB SI. Los factores que tienen una incidencia decisiva en la aplicación prescriptiva son:

- La necesidad de intervenir en todo el recorrido, ya que la escalera resultante pasa a ser en todos los casos protegida, por estar su altura de evacuación comprendida entre $14 \mathrm{~m}$ y $28 \mathrm{~m}$.

Tabla 2. Exigencias del DB SI en un levante.

\begin{tabular}{|c|c|c|c|c|c|}
\hline Exigencias Básicas & \multicolumn{4}{|c|}{ Características } & Zonas \\
\hline \multirow[t]{7}{*}{ Propagación interior } & Sectores & \multicolumn{3}{|c|}{$<2500 \mathrm{~m}^{2}$} & \\
\hline & $\begin{array}{l}\text { Resistencia en } \\
\text { elementos que separan } \\
\text { sectores } \\
\text { (Integridad y } \\
\text { Aislamiento) }\end{array}$ & \multicolumn{3}{|c|}{ EI 90} & Conjunto \\
\hline & \multirow[t]{5}{*}{ Reacción al fuego } & $\begin{array}{l}\text { Interior de las } \\
\text { viviendas }\end{array}$ & \multicolumn{2}{|l|}{ No se exige } & \\
\hline & & \multirow[t]{2}{*}{ Zonas ocupables } & Techos y paredes & C-s2, do & \multirow{4}{*}{ Recorrido de evacuación } \\
\hline & & & Suelos & EFL & \\
\hline & & \multirow[t]{2}{*}{ Escalera protegida } & Techos y paredes & B - s1, do & \\
\hline & & & Suelos & CFL - S1 & \\
\hline Propagación exterior & \multicolumn{4}{|c|}{ Condiciones de medianerías, fachadas y cubiertas (franjas >EI 60) } & Zona ampliada \\
\hline \multirow[t]{3}{*}{$\begin{array}{l}\text { Evacuación de } \\
\text { ocupantes }\end{array}$} & $\begin{array}{l}\text { Número de Salidas de } \\
\text { Planta }\end{array}$ & 1 & $\begin{array}{l}\text { Por altura de evact } \\
\text { de evacuación }\end{array}$ & ación y recorrido & Conjunto \\
\hline & \multirow[t]{2}{*}{ Tipo de escalera } & \multirow[t]{2}{*}{ Escalera protegida } & $\begin{array}{l}\text { Compartimentació } \\
\text { (EI 120, EI260-C5 }\end{array}$ & & \multirow[t]{2}{*}{$\begin{array}{l}\text { Recorrido de evacuación } \\
\text { del Conjunto }\end{array}$} \\
\hline & & & \multicolumn{2}{|c|}{$\begin{array}{l}\text { Protección frente al humo (ventana, } \\
\text { conductos, presurización) }\end{array}$} & \\
\hline Instalaciones de PCI & \multicolumn{4}{|c|}{ Extintores, alumbrado de emergencia, señalización } & $\begin{array}{l}\text { Recorrido de evacuación } \\
\text { del Conjunto }\end{array}$ \\
\hline $\begin{array}{l}\text { Intervención de los } \\
\text { bomberos }\end{array}$ & \multicolumn{4}{|c|}{ Condiciones de aproximación, entorno y accesibilidad por fachada } & Conjunto \\
\hline $\begin{array}{l}\text { Resistencia al fuego de } \\
\text { la Estructura }\end{array}$ & \multicolumn{2}{|c|}{ Resistencia (Capacidad portante) } & \multicolumn{2}{|c|}{ R 90} & Zona ampliada \\
\hline
\end{tabular}


- La exigencia de resistencia al fuego de la estructura, ya que, aunque se refiere a la zona ampliada, debería quedar condicionada, en buena medida, por la estructura existente en las plantas inferiores.

La escalera protegida, es por definición un recinto suficientemente seguro para la evacuación que debe reunir las siguientes condiciones:

- Disponer de elementos separadores EI 120

- Disponer, como máximo, de dos accesos por planta que se realicen a través de puertas EI26o $\mathrm{C}_{5}$ y desde espacios de circulación comunes y sin ocupación propia

- Disponer de protección frente al humo con ventilación natural mediante ventanas practicables de $1 \mathrm{~m}^{2}$ en cada planta, ventilación mediante conductos o sistema de presión diferencial.

La Figura 3 muestra las modificaciones que debe sufrir una escalera en un proyecto de levante con dos viviendas por planta. Es importante notar que la transformación más importante se produce al requerirse un espacio previo en cada acceso (desde un elemento de circulación sin ocupación propia), espacio que tiene un carácter funcional, pero que, básicamente, no aporta una mayor garantía a la compartimentación, que depende de la puerta de la escalera (EI2 6o C-5)

\section{ELECCIÓN DE MODELOS PARA LOS ANÁLISIS PRESTACIONALES}

Para poder establecer la fiabilidad de un diseño alternativo, es imprescindible poder comparar el nivel de riesgo del diseño propuesto con el que resulta de aplicar estrictamente las determinaciones de la regulación prescriptiva. Se considera muy importante también establecer el nivel de riesgo que tienen en la actualidad los edificios que son susceptibles de ser ampliados mediante levantes, ya que uno de los objetivos de la política reguladora de la edificación debe ser la reducción global del riesgo de incendio, riesgo muy importante, como se verá.

Se ha seleccionado, para los análisis prestacionales, un edificio real que reúne todas las características anteriormente descritas (Figura 4). Se trata de un edificio que, previamente, tiene un perfil de Sótano + Planta Baja + 5 Plantas de viviendas. Tras las obras de ampliación mediante levante queda con un perfil de Sótano + Planta Baja + 5 Plantas de viviendas +2 plantas de Levante. Las plantas de Levante cumplen la condición de que el acceso a la escalera se realiza a través de espacios previos de circulación, sin embargo las pantas existentes no lo hacen.

Es importante destacar que inicialmente en el estudio global se ha pretendido trabajar con tres edificios diferentes, que, respondiendo a la tipología descrita, presentan algunas diferencias en la distribución de patios respecto a las escaleras y a las viviendas. Al tratar de analizar por medios prestacionales la seguridad del recinto de escalera frente al humo, se desaconseja la utilización de ventilación natural mediante ventanas que la definición de escalera protegida ofrece. El hecho de que estas ventanas deban ser operadas de forma manual, así como la posibilidad de que la escalera pudiera quedar afectada por el humo, son algunas de las razones que nos conducen a ello.

Por otro lado, la dimensión del patio, en ningún caso llegará a ser suficiente para poder ventilarse la escalera, tras una operación de levante, de no contar con una dimensión mínima en proyección horizontal igual al círculo inscrito de diámetro $\mathrm{h} / 3$ ( $\mathrm{h}=$ altura patio). Por todo esto, se ha considerado centrar el esfuerzo en desarrollar todas las posibles simulaciones para un solo edificio (figura 4).
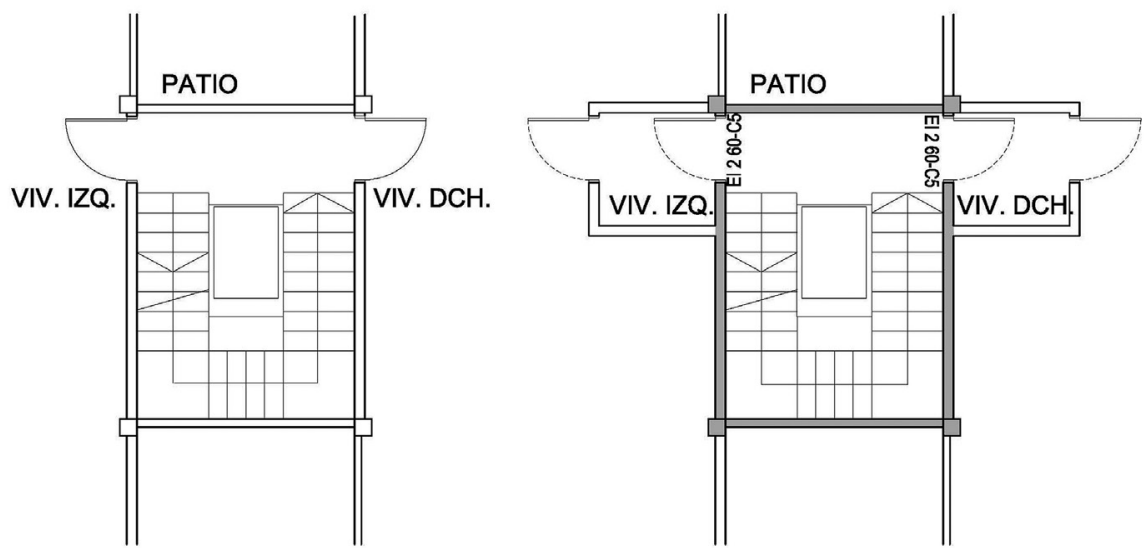

Figura 3. Escalera existente, sin proteger y Escalera Protegida, de acuerdo con las exigencias de la definición del DB SI.

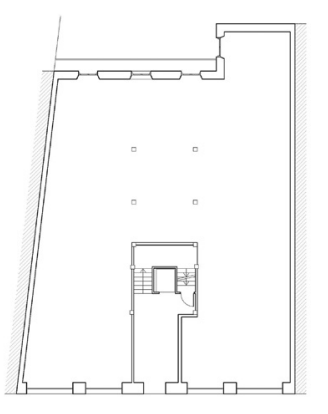

PLANTA BAJA

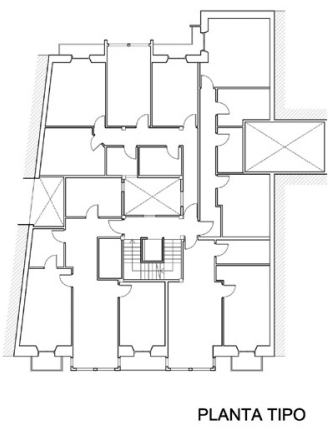

Figura 4. Plantas y Sección del edificio seleccionado.

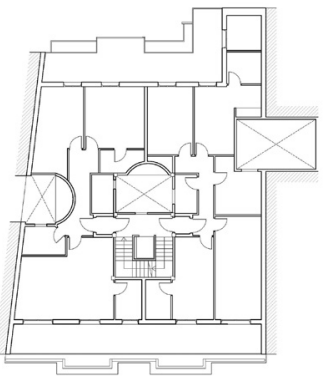

PLANTA

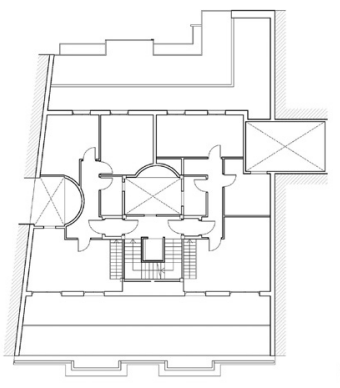

PLANTA 7

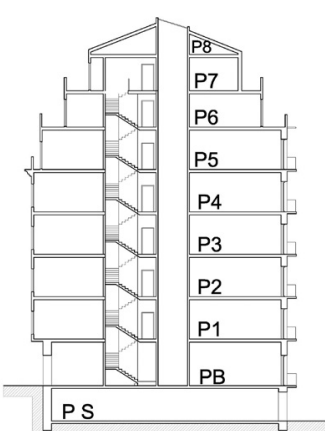


Se han desarrollado 6 casos para el edifico elegido (Tabla 3):

1. Diseño original, sin levante

2. Diseño prescriptivo de levante. Cumpliendo las condiciones del DB SI, con ventilación natural mediante conductos.

3. Diseño prescriptivo de levante. Cumpliendo las condiciones del DB SI, con ventilación mediante presurización

4. Diseño alternativo de levante. Sin espacio previo de circulación, puertas EI2 60 -C5en las viviendas y sistemas de PCI adicionales, con ventilación natural mediante conductos.

5. Diseño alternativo de levante. Sin espacio previo de circulación, puertas EI2 $60-\mathrm{C}_{5}$ en las viviendas y sistemas de PCI adicionales, con ventilación mediante presurización.

6. Diseño parcialmente prescriptivo. Cumple las condiciones de la escalera protegida del DB SI, pero únicamente en el levante, con ventilación natural mediante conductos. Este caso se ha considerado porque es la forma en la que se han abordado estas intervenciones a lo largo de algunos años.

\section{ANÁLISIS PRESTACIONAL CON MEREDICTE}

\subsection{Casos estudiados}

Se han estudiado los 6 casos descritos líneas arriba. Las comprensibles limitaciones del método impiden establecer una clara diferencia entre los diferentes sistemas de ventilación natural de la escalera, por conductos o a patio. Sin embargo ya se ha expuesto la inviabilidad de esta última en el análisis prestacional por simulación, por lo no se ha considerado.

\subsection{Metodología}

El método cuantifica el peligro potencial y lo contrasta con el nivel de protección en caso de incendio, de manera que se obtiene un nivel de riesgo del sector o del propio edificio. Contempla 73 coeficientes, divididos en dos bloques fundamentales:

1. "PP". PELIGRO POTENCIAL: Determinado por 28 coeficientes de valoración del Peligro.

2. "NPE" NIVEL DE PROTECCIÓN del EDIFICIO: Determinado por 45 coeficientes de valoración del Grado de Protección

De la relación entre ambas magnitudes, se obtiene el NIVEL DE RIESGO DEL EDIFICIO, definido por la expresión [1]:

$$
N R E=P P / N P E
$$

\subsection{Resultados}

La Tabla 4 recoge el desarrollo y resultado del cálculo del PELIGRO POTENCIAL, que queda definido por la expresión [2]:

$$
P P=A S^{*}\left(O, 4^{*} T+O, 3^{*} \mathrm{CO}+O, 3^{*} \mathrm{CA}\right)
$$

Los peligros potenciales valoran el Aforo del Sector(AS), el Tetraedro del fuego(T), y las características de los Ocupantes(CO) y Arquitectónicas del edificio(CA). Cada uno de ellos tiene un peso específico y a su vez éste, está ponderado por diferentes coeficientes, la mayoría de ellos ligados al uso, en nuestro caso vivienda, y por tanto no presentan variación en los diferentes supuestos, pero sí son de destacar los siguientes:

- Tetraedro del Fuego(T). Es significativo el aumento de peligro que supone la Energía de Activación, en el edificio existente, debido a la consideración que se hace del estado de las instalaciones eléctricas y del posible uso de sistemas de calefacción de llama abierta. Los locales de riesgo considerados en el DBSI, como los cuartos de contadores eléctricos o los trasteros, ponderan muy negativamente si no cuentan con las medidas de protección previstas en el DB-SI 1.2.

Combustible (TC). Refleja lo que supone la carga de fuego inmobiliaria, disminuyendo en un $12 \%$ el peligro potencial en edificios de estructura de hormigón.

- Reacción en Cadena (TCR). Pondera cómo influye la configuración arquitectónica, ya que incide en la reacción en cadena del fuego y por tanto en su virulencia en la propagación. Sin embargo, indicar que los supuestos que aporta el método no se ajustan a los escenarios de los edificios de vivienda.

- Características Arquitectónicas. Ponderan de manera diferente aumentando la influencia en el peligro potencial la mayor altura de evacuación y la mayor superficie del sector en las hipótesis del edificio con levante frente al existente, si bien en nuestro caso son equivalentes.

La Tabla 5 recoge el desarrollo y resultado del cálculo del NIVEL DE PROTECCIÓN EDIFICATORIA, que queda definido por la expresión [3]:

$$
\text { [3] } N P E=R F E x(O, 24 P+O, 4 O E O+O, 24 I P C I+O, 12 I B)
$$

y el resultado del NIVEL DE RIESGO DEL EDIFICIO.

Del Nivel de Protección Edificatoria, destacamos:

- Resistencia al Fuego de la Estructura(RFE): Reduce un $80 \%$ el nivel de protección de NO conocerse ésta. Teniendo

\begin{tabular}{|c|c|c|c|c|c|c|c|}
\hline & \multicolumn{2}{|c|}{ Tipo de diseño } & \multicolumn{2}{|c|}{ Características escalera } & \multirow{2}{*}{\begin{tabular}{|l|} 
Medidas adicionales \\
- Puertas EI2 6o $\mathrm{C} 5$ \\
en viviendas \\
- Detección y alarma \\
\end{tabular}} & \multicolumn{2}{|c|}{ Protección frente al humo } \\
\hline Diseño & Prescriptivo & Prestacional & $\begin{array}{l}\text { Protegida con } \\
\text { espacio de } \\
\text { circulación previo }\end{array}$ & $\begin{array}{l}\text { Sin espacio } \\
\text { de circulación } \\
\text { previo }\end{array}$ & & $\begin{array}{l}\text { Ventilación natural } \\
\text { con conductos }\end{array}$ & Presurización \\
\hline 1 & \multicolumn{7}{|c|}{ Edificio existente } \\
\hline 2 & $\mathrm{X}$ & & $\mathrm{X}$ & & & $\mathrm{X}$ & \\
\hline 3 & $\mathrm{X}$ & & $\mathrm{X}$ & & $\mathrm{X}$ & & $\mathrm{X}$ \\
\hline 4 & & $\mathrm{X}$ & & $\mathrm{X}$ & $\mathrm{X}$ & $\mathrm{X}$ & \\
\hline 5 & & $\mathrm{X}$ & & $\mathrm{X}$ & $\mathrm{X}$ & & $\mathrm{X}$ \\
\hline 6 & \multicolumn{2}{|c|}{ Parcialmente } & Plantas ampliadas & $\begin{array}{l}\text { Plantas } \\
\text { existentes }\end{array}$ & & $\mathrm{X}$ & \\
\hline
\end{tabular}
en cuenta que estamos actuando sobre un parque existente

Tabla 3. Esquema de las características de los seis casos a análisis. 
Tabla 4. Resumen de aplicación del método MEREDICTE a los seis casos a análisis.

\begin{tabular}{|c|c|c|c|c|c|c|}
\hline $\begin{array}{c}\text { EDIFICIO DE VIVIENDAS } \\
\text { EXISTENTE TIPOLOGÍA ESCALERA } \\
\text { A PATIO }\end{array}$ & $\mathrm{VIV}=\mathrm{S}+\mathrm{B}+\mathbf{5}$ & $\begin{array}{c}\text { VIV }=\mathbf{S}+\mathbf{B}+5^{+} \\
\text {2lev } \mathbf{C T E} \text {. } \\
\text { Ventilación } \\
\text { conductos }\end{array}$ & $\begin{array}{c}\text { VIV }=\mathrm{S}+\mathrm{B}+5^{+} \\
\text {2lev CTE. } \\
\text { Ventilación } \\
\text { Presurización }\end{array}$ & $\begin{array}{c}\text { VIV }=\mathrm{B}+5^{+} \\
\text {2lev CTE, SIN } \\
\text { ESCALERA } \\
\text { PROTEGIDA, } \\
\text { Ventilación } \\
\text { Conductos }\end{array}$ & $\begin{array}{c}\text { VIV }=\mathrm{B}+5^{+} \\
\text {2lev CTE, SIN } \\
\text { ESCALERA } \\
\text { PROTEGIDA, } \\
\text { Ventilación } \\
\text { Presurización }\end{array}$ & $\begin{array}{c}\mathrm{VIV}=\mathrm{S}+\mathrm{B}+5+ \\
\text { 2lev CTE solo } \\
\text { en levante. } \\
\text { Ventilación } \\
\text { conductos }\end{array}$ \\
\hline h libre (m) & 3,41 & 3,41 y 2,7 & 3,41 y 2,7 & 3,41 y 2,7 & 3,41 y 2,7 & 3,41 y 2,7 \\
\hline h-evacuación (m) & 18,38 & $\mathbf{2 4 , 1}$ & $\mathbf{2 4 , 1}$ & $\mathbf{2 4 , 1}$ & $\mathbf{2 4 , 1}$ & $\mathbf{2 4 , 1}$ \\
\hline Sup. Const. $\left(\mathrm{m}^{2}\right)$ & 1.627 & 1.784 & 1.784 & 1.784 & 1.784 & 1.784 \\
\hline \multirow[t]{2}{*}{ Aforo (personas) } & 70 & 91 & 91 & 91 & 91 & 91 \\
\hline & Caso.1 & Caso.2 & Caso.3 & Caso.4 & Caso.5 & Caso.6 \\
\hline \multirow[t]{2}{*}{ PP = PELIGRO POTENCIAL } & MUY ALTO & ALTO & ALTO & ALTO & ALTO & ALTO \\
\hline & 11,18 & 3,34 & 3,34 & 3,34 & 3,34 & $\mathbf{4 , 5 7}$ \\
\hline
\end{tabular}

\begin{tabular}{|l|l|c|c|c|c|c|c|}
\hline AS(1) & Aforo Sector & 1 & 1 & 1 & & 1 & \\
\hline 40\% T (13) & Tetraedro del fuego & $\mathbf{2 2 , 8 0}$ & 3,19 & 3,19 & 3,19 & 3,19 & 6,27 \\
\hline TC & (Combustible) $=$ & 2,42 & 2,42 & 2,42 & 2,42 & 2,42 & 2,42 \\
\hline TCM & (Comburente) & 1 & 1 & 1 & 1 & 1 & 1 \\
\hline TEA & (Energía de activación) $=$ & $\mathbf{7 , 2 5}$ & 1,1 & 1,1 & 1,1 & 1,1 & 1,99 \\
\hline & ea2 = Riesgos especiales & 4 & 1,1 & 1,1 & 1,1 & 1,1 & 1,1 \\
\hline & ea4 = Instala eléctrica & 1,45 & 1 & 1 & 1 & 1 & 1,45 \\
\hline & ea5 = Calefacción & 1,25 & 1 & 1 & 1 & 1 & 1,25 \\
\hline TRC & (Reacción en cadena) $=$ & 1,3 & 1,2 & 1,2 & 1,2 & 1,2 & 1,3 \\
\hline $\mathbf{3 0 \% ~ C O ( 7 ) ~}$ & Caract. Ocupantes & 1,04 & 1,04 & 1,04 & 1,04 & 1,04 & 1,04 \\
\hline $\mathbf{3 0 \%}$ CA(7) & Caráct. Arquitecton. & 5,82 & 5,82 & 5,82 & 5,82 & 5,82 & 5,82 \\
\hline & Ca1 = Sup. sector incend. & 1,2 & 1,2 & 1,2 & 1,2 & 1,2 & 1,2 \\
\hline & Ca2 =Cof. altura evac s/r & 2,2 & 2,2 & 2,2 & 2,2 & 2,2 & 2,2 \\
\hline & Ca3 =Cof. Núm. Plan. b/r & 1,4 & 1,4 & 1,4 & 1,4 & 1,4 & 1,4 \\
\hline
\end{tabular}

y fundamentalmente de estructura de madera, anterior a la legislación de protección contra incendios, consideramos NO conocerla: coeficiente 0,2 en el caso 1 . De conocerse su comportamiento y ser ajustado a norma, es susceptible de imputar un coeficiente de 1,15, extremo este, a ponderar según sean las condiciones de adecuación efectiva. Propagación del Incendio(P). La falta de compartimentación de sectores en los edificios existentes con respecto a sus locales comerciales y la posible propagación exterior que pueda darse, penalizan en la hipótesis de estado actual.

- Evacuación de Ocupantes(EO). Ponderan de manera dispar las diferentes hipótesis en relación al tipo de salidas verticales y al tipo de ventilación de la escalera.

- Instalaciones de Protección Contra el Incendio(IPCI). El cumplimiento del DB SI supone dotar a las intervenciones de alumbrado de emergencia y de extintores de incendio, además de señalización. Además, si como medidas compensatorias, se propone la detección y alarma, el nivel de protección aumenta; toda vez que el incendio puede ser detectado con mayor celeridad y evitar así su propagación.

- Manual de Autoprotección. El MEREDICTE pondera aumentando un 20\% el Nivel de Protección Edificatoria, siempre que se disponga de éste; si de antemano no es prescriptivo. En el caso de viviendas no lo es.

\section{ANÁLISIS PRESTACIONAL DE RIESGOS CUANTITATIVO CON SIMULACIÓN}

\subsection{Casos estudiados}

El análisis compara todos los casos anteriormente definidos. Las herramientas que se utilizan son FDS (Fire Dynamics Si- mulator, Pyrosim), para la modelización del incendio y movimiento de humos y PATHFINDER, para la modelización de la evacuación de las personas.

\subsection{Metodología}

El nivel de riesgo se define por la expresión [4]:

\section{[4] Nivel de riesgo $=\sum$ Probabilidad $x$ Consecuencia}

Para el cálculo de las probabilidades, se utiliza un sistema de árboles de eventos, tal y como se representa en la Figura 5 .

En el diagrama, F, es la probabilidad de que ocurra el evento inicial, Ps1 es la probabilidad de que ocurra el evento nodal 1 y PF1 es la probabilidad de que este no ocurra (Ps1 y PF1 deben sumar 1). PS2 y PF2 representan las probabilidades para el evento nodal 2. F1, F2, F3 y F4 son las probabilidades de que ocurra cada resultado final respectivamente.

Se han elaborado, para cada uno de los diseños definidos, árboles de probabilidad con 5 parámetros (ignición, estado de los ocupantes, detección y alarma, compartimentación y ventilación). Su descripción y las probabilidades de cada evento se reflejan en la Tabla 6.

Para el cálculo de las consecuencias, se utiliza el valor de las personas afectadas por el humo en los diferentes escenarios para las diferentes simulaciones. Se ha definido como persona afectada por el humo, aquella que, para evacuar, ha de cruzar una zona en la que la visibilidad es menor de $3 \mathrm{~m}$ (16). 
Tabla 5. Resumen de aplicación del método MEREDICTE a los seis casos a análisis.

\begin{tabular}{|c|c|c|c|c|c|c|c|}
\hline \multicolumn{2}{|c|}{$\begin{array}{l}\text { NPG = NIVEL PROTEC. } \\
\text { EDIFICATORIO GLOBAL }\end{array}$} & INSIGNIFICANTE & ALTO & ALTO & ALTO & ALTO & BAJO \\
\hline \multicolumn{2}{|r|}{ NPG $=$ NPA $\times$ NPE } & $\mathbf{0 , 1 0}$ & 2,18 & $\mathbf{2 , 5 7}$ & 2,39 & 2,72 & $\mathbf{0 , 9 2}$ \\
\hline \multirow{2}{*}{\multicolumn{2}{|c|}{$\begin{array}{r}\mathrm{NPE}=\mathrm{RFE} x \\
(0,24 \mathrm{P}+0,40 E O+0,24 \mathrm{IPCI}+0,12 \mathrm{IB}) \\
\end{array}$}} & $\mathbf{0 , 1 0}$ & 2,18 & 3,63 & $\mathbf{2 , 0 0}$ & $\mathbf{2 , 2 7}$ & $\mathbf{0 , 9 2}$ \\
\hline & & Caso.1 & Caso.2 & Caso.3 & Caso.4 & Caso.5 & Caso.6 \\
\hline NPA & $\begin{array}{r}\text { NIVEL PLAN } \\
\text { AUTOPROTECCIÓN }\end{array}$ & 1 & $\mathbf{1}$ & $\mathbf{1}$ & 1,2 & 1,2 & $\mathbf{1}$ \\
\hline
\end{tabular}

\begin{tabular}{|c|c|c|c|c|c|c|c|}
\hline $\mathbf{R F E}(1)$ & $\begin{array}{l}\text { Resistencia al Fuego } \\
\text { Estructura }\end{array}$ & $\mathbf{0 , 2}$ & 1,15 & 1,15 & 1,15 & 1,15 & 1,15 \\
\hline $24 \% \mathbf{P}(10)$ & Propagación del incendio & 0,48 & 0,86 & 0,86 & 0,86 & 0,86 & 0,86 \\
\hline \multirow[t]{3}{*}{ PI1 } & (Compart. en Sectores In.) & 1 & 1,44 & 1,44 & 1,44 & 1,44 & 1,44 \\
\hline & 1.1 RF pared, techo sector. & 1 & 1,2 & 1,2 & 1,2 & 1,2 & 1,2 \\
\hline & 1.5 Paso instala. Compart. & 1 & 1,2 & 1,2 & 1,2 & 1,2 & 1,2 \\
\hline PI2 & (Grado reacción fuego) & 0,6 & 0,6 & 0,6 & 0,6 & 0,6 & 0,6 \\
\hline $\mathrm{PE}$ & (Propagación Exterior) & $\mathbf{0 , 8}$ & 1 & 1 & 1 & 1 & 1 \\
\hline PE1 & Propag. fachada y cubierta. & 0,8 & 1 & 1 & 1 & 1 & 1 \\
\hline \multirow[t]{6}{*}{ 40\% EO(15) } & Evacuación Ocupantes & $\mathbf{0 , 5 1}$ & 3,39 & 4,23 & 2,37 & 2,96 & 0,64 \\
\hline & 4.3 Tipo salidas verticales & 0,6 & 2 & 2 & 1,4 & 1,4 & 0,6 \\
\hline & 4.4 Contin.de las escaleras & 1 & 1,2 & 1,2 & 1,2 & 1,2 & 1 \\
\hline & 4.5 Ventila. Escal. Proteg. & 1 & 1 & 1,25 & 1 & 1,25 & 1 \\
\hline & 6.Señaliz. medios evacua. & 0,6 & 1 & 1 & 1 & 1 & 1 \\
\hline & 7. Control Humo Incendio & 1 & 1 & 1 & 1 & 1 & 1 \\
\hline 24\% IPCI(15) & Inst. Protec. Incendios & $\mathbf{0 , 1 3}$ & 0,69 & 0,69 & 1,72 & 1,72 & 0,69 \\
\hline ipci1.1 & 1.1 Detección de incendios & 0,7 & 0,7 & 0,7 & 1 & 1 & 0,7 \\
\hline ipci1.2 & 1.2 Tipo de detección & 1 & 1 & 1 & 1,5 & 1,5 & 1 \\
\hline ipci1.3 & 1.3 Identifica. detector & 1 & 1 & 1 & 1,1 & 1,1 & 1 \\
\hline ipci1.4 & 1.4 Sist. alarma incendios & 1 & 1 & 1 & 1,05 & 1,05 & 1 \\
\hline Ipci2.1 & 2.1 Extintores & 0,7 & 1 & 1 & 1 & 1 & 1 \\
\hline Ipci4.1 & 4.1 Señaliz. medios evacua. & 0,9 & 1 & 1 & 1 & 1 & 1 \\
\hline Ipci4.3 & Alumbrado de Emergencia & 0,3 & 1 & 1 & 1 & 1 & 1 \\
\hline \multirow[t]{2}{*}{ 12\% IB(3) } & Interven. Bomberos & 1,4 & 1,4 & 1,4 & 1,4 & 1,4 & 1,4 \\
\hline & & Caso.1 & Caso.2 & Caso.3 & Caso.4 & Caso.5 & Caso.6 \\
\hline \multicolumn{2}{|c|}{ NIVEL DE RIESGO DEL EDIFICIO } & CATASTROFICO & \begin{tabular}{|c|} 
MUY \\
ELEVADO
\end{tabular} & ELEVADO & ELEVADO & ELEVADO & GRAVE \\
\hline \multicolumn{2}{|c|}{ NRE $=$ PP / NPG } & 107,95 & $\mathbf{1 , 5 3}$ & 1,30 & 1,39 & 1,23 & 4,99 \\
\hline
\end{tabular}

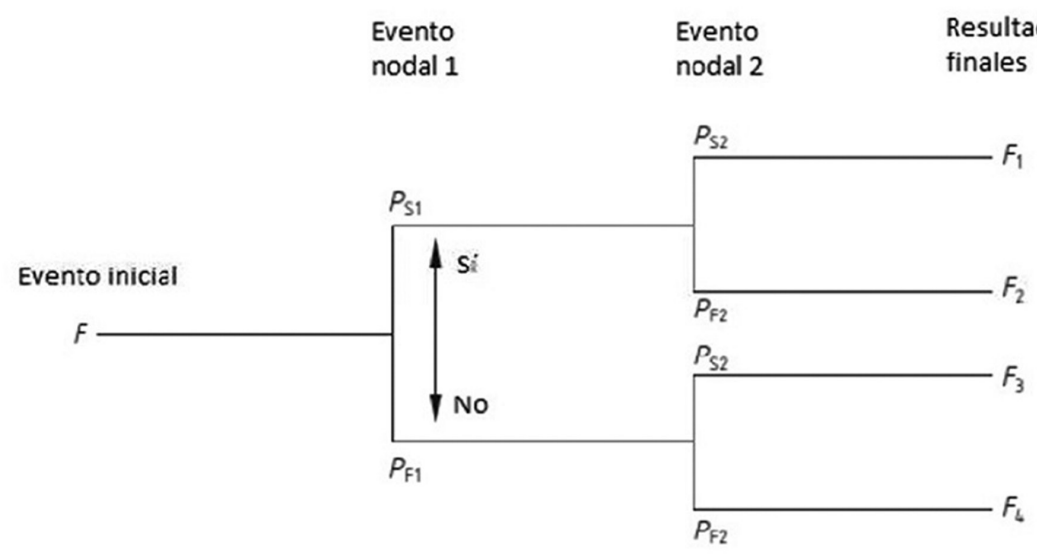

Figura 5. Esquema de árbol de eventos simple. PD 7974-7.

Para todas las simulaciones se ha utilizado un incendio en un salón de una de las viviendas de la planta primera del edificio. El incendio comienza en una mesa. Tiene un coeficiente de crecimiento lento, con valor $0,003 \mathrm{~kW} / \mathrm{s}^{2}$ durante $20 \mathrm{mi}-$ nutos. La tasa de liberación de calor que alcanza en los 20 minutos es de $4320 \mathrm{~kW}$ (17). Se ha desarrollado de tal manera que se garantiza una producción de humos relativamente alta durante todo el tiempo de simulación.

La caracterización y determinación del comportamiento de los ocupantes se puede simplificar en premovimiento y movimiento. Como el tiempo de premovimiento es muy irregular en vi- 
Tabla 6. Probabilidades de cada evento.

\begin{tabular}{|l|l|l|c|c|}
\hline \multicolumn{1}{|c|}{ Evento } & \multicolumn{1}{|c|}{ Descripción } & \multicolumn{1}{c|}{ Comentarios } & SI & NO \\
\hline Ignición & Probabilidad de ignición & & $\mathbf{1}$ & - \\
\hline Estado de los ocupantes & ¿Están despiertos? & & $\mathbf{0 , 6 7}$ & $\mathbf{0 , 3 3}$ \\
\hline \multirow{2}{*}{ Detección y Alarma } & \multirow{2}{*}{\begin{tabular}{l} 
Sin detección y alarma \\
\cline { 2 - 5 }
\end{tabular}} & $\begin{array}{l}\text { Con detección y alarma } \\
\text { Detector de humo doméstico } \\
\text { Probabilidad detección: 0,75 (18) } \\
\text { Probabilidad alarma: o,95(18) }\end{array}$ & $\mathbf{0 , 7 1 2 5}$ & $\mathbf{0 , 2 8 7 5}$ \\
\hline Compartimentación & ¿Funciona el sistema? & Resistencia de las puertasEI260-C5 (18) & $\mathbf{0 , 8}$ & $\mathbf{0 , 2}$ \\
\hline Ventilación & ¿Funciona el sistema? & Por conductos: 1 & $\mathbf{1}$ & - \\
\cline { 2 - 6 } & Presurización:0,9 (18) & $\mathbf{0 , 9}$ & $\mathbf{0 , 1}$ \\
\hline
\end{tabular}

viendas, se ha optado por un incendio que crea un ambiente difícil de movimiento (bajada de visibilidad) dentro de la escalera común durante la fase de evacuación del edificio. Un incendio de crecimiento más rápido probablemente sería más ventajoso para los ocupantes que evacuan por la escalera ya que gran parte del humo producido se ventilaría directamente al exterior. En cualquier caso, se trata de un análisis comparativo.

Para los escenarios en los que hay detección automática, el tiempo de detección se ha evaluado mediante los resultados de las simulaciones de movimiento de humo. En los modelos de simulación, se ha establecido un sistema de detección puntual, y se ha determinado que el tiempo de detección y alarma es de 40 segundos.

Se han introducido 4 tipos de ocupantes en el modelo. Un $25 \%$ hombres adultos, un $25 \%$ mujeres adultas, un $28,5 \%$ ancianos y un $21,5 \%$ niños. Se han establecido velocidades mínimas y máximas para cada uno de estos tipos: 1,10 y 1,60, 1,05 y 1,4, o,5 y 1,1 y o,6 y 1,2 m/s respectivamente.

El número de ocupantes, 70 personas en el caso 1 y 91 personas en los casos 2 a 6 , se ha establecido de acuerdo con las densidades de ocupación del DB SI3.

Del análisis de los árboles de eventos en los 6 casos estudiados, resultan 11 consecuencias diferentes ( $\mathrm{C} 1$ a C11). Estas se diferencian en el número de ocupantes (hay levante o no), en si están dormidos o no y en la presencia o no de sistemas de detección y alarma. En definitiva, 6 simulaciones (Figura 6) para 10 consecuencias, ya que la consecuencia $\mathrm{C} 1$ representa el funcionamiento de todos los sistemas y, por tanto, no hay afectados por el humo.

Para la modelización del movimiento de evacuación con Pathfinder se ha utilizado el modo que implementa los con- ceptos manejados en el SFPE Handbook of Fire Protection Engineering (19). Este es un modelo de flujo donde la velocidad de paso de los ocupantes está determinada por la densidad de ocupación dentro de cada habitación, y el flujo de ocupantes a través de las puertas está controlado por el ancho de dichas puertas.

\subsection{Resultados}

Se han realizado 10 simulaciones de incendio y de evacuación como resultado de los árboles de eventos. Para cada una de ellas se han calculado las personas afectadas por el humo (consecuencias). El nivel de riesgo para cada uno de los casos se recoge en la Tabla 7 .

La gráfica de barras de la Figura 7 presenta la comparativa del nivel de riesgo obtenido para cada uno de los casos estudiados y la Tabla 8 relaciona los niveles de riesgo obtenidos con cada uno de los casos estudiados y las medidas utilizadas

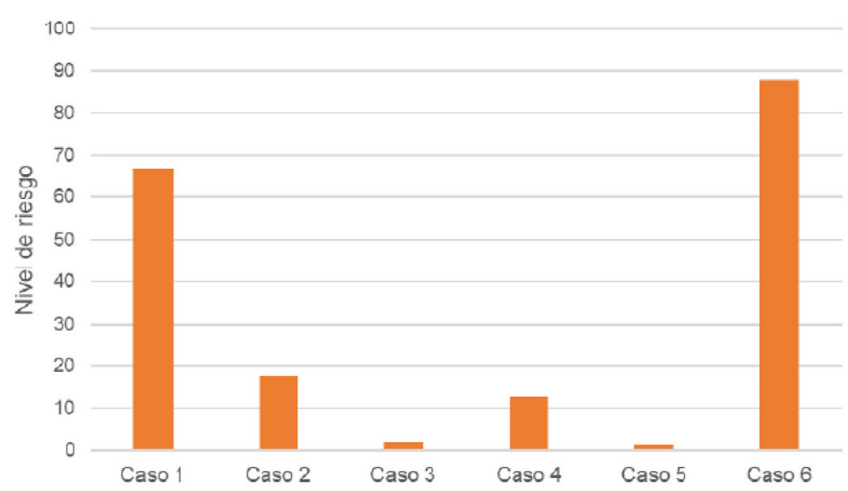

Figura 7. Nivel de riesgo para cada uno de los casos estudiados.
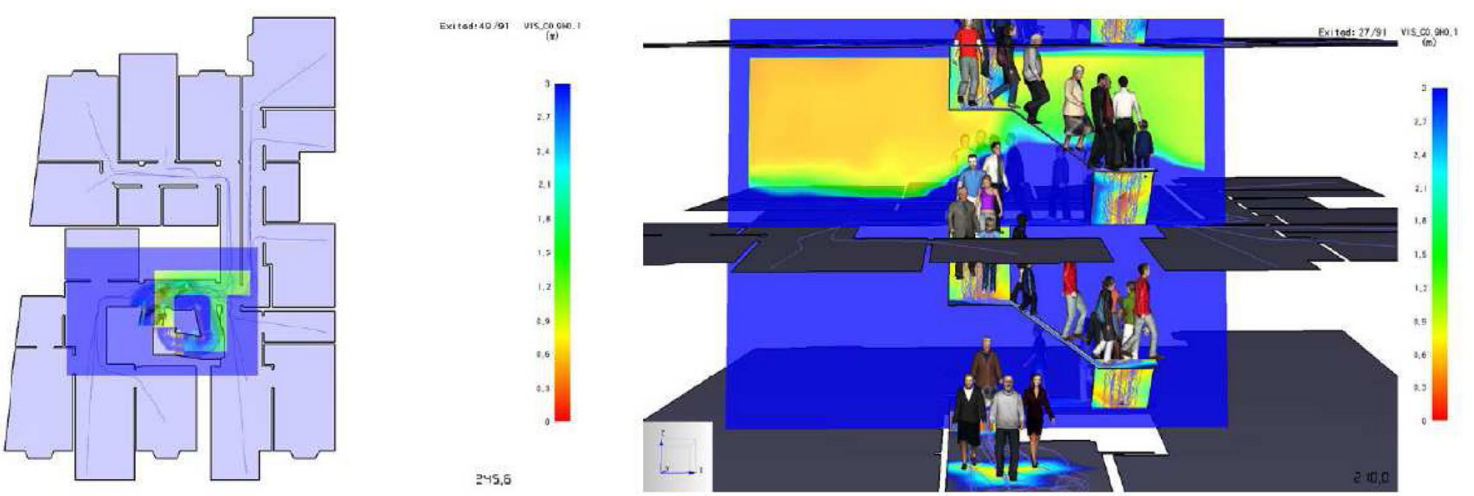

Figura 6. Simulaciones de incendio y evacuación para el caso C2. 
Tabla 7. Consecuencias y número de personas afectadas.

\begin{tabular}{|c|c|c|c|c|c|c|c|}
\hline \multirow[b]{2}{*}{$\mathbf{C i}$} & \multirow[b]{2}{*}{ Levante } & \multicolumn{4}{|c|}{ Eventos dada la ignición } & \multirow[b]{2}{*}{ Valor } & \multirow[b]{2}{*}{ Casos afectados } \\
\hline & & $\begin{array}{l}\text { Ocupantes } \\
\text { despiertos }\end{array}$ & Detección & Compartimentación & Ventilación & & \\
\hline $\mathrm{C} 1$ & $\mathrm{Si}$ & Irrelevante & Irrelevante & $\mathrm{Si}$ & Si(presurización) & o & $2,3,4$ y 5 \\
\hline $\mathrm{C} 2$ & $\mathrm{Si}$ & $\mathrm{Si}$ & $\mathrm{Si}$ & No & Si(conductos) & 42 & 4 \\
\hline $\mathrm{C}_{3}$ & $\mathrm{Si}$ & $\mathrm{Si}$ & $\mathrm{Si}$ & No & No(presurización) & 37 & 5 \\
\hline $\mathrm{C}_{4}$ & $\mathrm{Si}$ & $\mathrm{Si}$ & No & No & Si(conductos) & 88 & 2,4 y 6 \\
\hline $\mathrm{C}_{5}$ & $\mathrm{Si}$ & $\mathrm{Si}$ & No & No & No(presurización) & 88 & 3 у 5 \\
\hline $\mathrm{C} 6$ & $\mathrm{Si}$ & No & $\mathrm{Si}$ & No & Si(conductos) & 81 & 4 \\
\hline $\mathrm{C}_{7}$ & $\mathrm{Si}$ & No & $\mathrm{Si}$ & No & No(presurización) & 81 & 5 \\
\hline $\mathrm{C} 8$ & $\mathrm{Si}$ & No & No & No & Si(conductos) & 88 & 2,4 y 6 \\
\hline $\mathrm{C} 9$ & $\mathrm{Si}$ & No & No & No & No(presurización) & 88 & 3 у 5 \\
\hline C10 & No & $\mathrm{Si}$ & No & No & No dispone & 67 & 1 \\
\hline $\mathrm{C} 11$ & No & No & No & No & No dispone & 67 & 1 \\
\hline
\end{tabular}

Tabla 8. Nivel de riesgo para cada uno de los casos.

\begin{tabular}{|c|c|c|c|c|c|c|c|c|}
\hline & \multicolumn{3}{|c|}{ Medidas de PCI } & \multicolumn{2}{|c|}{ Protección frente al humo en escalera } & \multirow[b]{2}{*}{$\mathbf{P}$} & \multirow[b]{2}{*}{$\mathbf{C}$} & \multirow[b]{2}{*}{$\mathbf{R}$} \\
\hline & $\begin{array}{c}\text { Escalera } \\
\text { Protegida }\end{array}$ & $\begin{array}{l}\text { Detección } \\
\text { y alarma }\end{array}$ & $\begin{array}{l}\text { Puerta resistente cierre } \\
\text { automático viviendas }\end{array}$ & $\begin{array}{c}\text { Ventilación natural } \\
\text { conductos }\end{array}$ & Presurización & & & \\
\hline \multirow{2}{*}{ Caso 1} & \multirow{2}{*}{\multicolumn{5}{|c|}{ Carece de cualquier medida }} & $67 \%$ & 67 & \multirow{2}{*}{67,0} \\
\hline & & & & & & $33 \%$ & 81 & \\
\hline \multirow{2}{*}{ Caso2 } & \multirow{2}{*}{$\mathrm{Si}$} & \multirow{2}{*}{ - } & \multirow{2}{*}{-} & \multirow{2}{*}{$\mathrm{Si}$} & \multirow{2}{*}{-} & $13,4 \%$ & 88 & \multirow{2}{*}{17,6} \\
\hline & & & & & & $6,6 \%$ & 88 & \\
\hline \multirow{2}{*}{ Caso 3} & \multirow{2}{*}{$\mathrm{Si}$} & \multirow{2}{*}{ - } & \multirow{2}{*}{-} & \multirow{2}{*}{-} & \multirow{2}{*}{$\mathrm{Si}$} & $1,34 \%$ & 88 & \multirow{2}{*}{1,8} \\
\hline & & & & & & $0,66 \%$ & 88 & \\
\hline \multirow{4}{*}{ Caso 4} & \multirow{4}{*}{-} & \multirow{4}{*}{$\mathrm{Si}$} & \multirow{4}{*}{$\mathrm{Si}$} & \multirow{4}{*}{$\mathrm{Si}$} & \multirow{4}{*}{-} & $9,5 \%$ & 42 & \multirow{4}{*}{12,9} \\
\hline & & & & & & $3,8 \%$ & 88 & \\
\hline & & & & & & $4,7 \%$ & 81 & \\
\hline & & & & & & $1,9 \%$ & 88 & \\
\hline \multirow{4}{*}{ Caso 5} & \multirow{4}{*}{-} & \multirow{4}{*}{$\mathrm{Si}$} & \multirow{4}{*}{$\mathrm{Si}$} & \multirow{4}{*}{-} & \multirow{4}{*}{$\mathrm{Si}$} & $0,95 \%$ & 37 & \\
\hline & & & & & & $0,38 \%$ & 88 & 12 \\
\hline & & & & & & $0,47 \%$ & 81 & 1,2 \\
\hline & & & & & & $0,19 \%$ & 88 & \\
\hline Caso 6 & Parcial & _ & _- & $\mathrm{Si}$ & _ & $67 \%$ & 88 & 880 \\
\hline & & - & & & - & $33 \%$ & 88 & \\
\hline
\end{tabular}

\section{DISCUSIÓN Y TRABAJO FUTURO}

Aplicado el método MEREDICTE y la simulación (FDS Y PATHFINDER), a los seis casos podemos señalar que:

Ambos métodos muestran que el riesgo de incendio, tanto en los edificios existentes $(\mathrm{Rs}=67,0, \mathrm{Rm}=107,95)$, como en los ampliados en los que la protección se limita a la zona elevada $(\mathrm{Rs}=88, \mathrm{Rm}=4,99)$, es desorbitado. La comparación entre los diseños prescriptivos y los alternativos es favorable a estos últimos al incrementarse en estos últimos las medidas de protección activa, tanto para la ventilación natural por conductos $(\mathrm{Rs}=12,9$ frente a 17,6), $(\mathrm{Rm}=1,39$ frente a 1,53$)$ como para la presurización $(R s=1,2$ frente a 1,8), $(R m=1,23$ frente a 1,3).

El orden de magnitud de los resultados obtenidos con ambos análisis es bastante coherente.

- El riesgo de los edificios que no se rehabilitan se dispara respecto a las opciones de levante. Este, está asociado a una gran incertidumbre por la muy variable condición del parque edificado, pero el resultado es muy coherente con las estadísticas de incendio.
- La reducción del riesgo que se obtiene con los diseños alternativos respecto a los que cumplen estrictamente la norma prescriptiva, en el caso de la ventilación natural por conductos oscila entre el $5 \%$ y el $27 \%$ y en el caso de la presurización entre el 10\% y el 33\%. Esta reducción se basa fundamentalmente en la implementación de protección activa.

- La estructura de la parte preexistente debe ser objeto de análisis en cada caso concreto. De la lectura del CTE y de los Comentarios del Ministerio de Fomento no puede extraerse una conclusión definitiva sobre el cumplimiento obligado de su resistencia. Se debe tener en cuenta, además, que se trata de intervenciones al amparo de la Ley 8/2013, por lo que se debería procurar en la misma el mayor grado posible de adecuación efectiva.

La línea de trabajo seguida aconseja implementar y/o mejorar métodos de evaluación que permitan una mayor precisión a la hora de abordar el riesgo de incendio en edificios de vivienda. Para dar validez a los resultados es imprescindible establecer algunas limitaciones. Es importante no superar en ningún caso la altura de evacuación de $28 \mathrm{~m}$, garantizar la 
compartimentación mediante sistemas de cierre de puertas fiables (como pueden ser los que permiten la función de giro libre sin la resistencia del cierrapuertas, que se activan en caso de incendio, regresando la puerta a la posición de cerrada) y establecer un adecuado programa de mantenimiento y autoprotección.

\section{CONCLUSIONES}

- La adición limitada de plantas en edificios de viviendas existentes constituye una operación clave con el objeto de densificar el centro de las ciudades. Debe generar, además, un beneficio económico que repercuta en una mejora sustancial de las prestaciones de los edificios, entre las que caben destacar el desempeño energético y la seguridad en caso de incendio.

- La aplicación del documento prescriptivo de Seguridad en Caso de Incendio del CTE, Documento Básico SI, imposibilita, en la mayoría de los casos, la ejecución de proyectos de levantes. La razón fundamental es la exigencia de que la escalera protegida, de acuerdo con su definición, prescribe que sus accesos se realicen desde espacios "de circulación comunes y sin ocupación propia”. Estos espacios previos a la escalera, sin embargo, no aportan, por sí mismos, una mejora sustancial de las condiciones de seguridad del edificio. El origen de esa exigencia hay que buscarlo en el de- sarrollo histórico de la escalera protegida en los códigos de edificación, fundamentalmente en los casos de ocupaciones importantes.

- El análisis de riesgo cuantitativo efectuado, tanto con el programa MEREDICTE, documento reconocido del CTE, como con herramientas de simulación (FDS y PATHFINDER), muestra que, algunos diseños que no cumplen estrictamente las condiciones del documento prescriptivo, pueden tener un riesgo de incendio menor que el que se obtendría mediante su aplicación. Los resultados muestran coherencia y evidencian la importancia de la protección activa.

- Cualquier solución prestacional debe ir acompañada de todas las medidas que garanticen su correcta implantación. En el caso de los edificios de viviendas, son imprescindibles el mantenimiento de los sistemas y la autoprotección.

\section{FINANCIACIÓN}

El presente trabajo, que está siendo desarrollado por la Delegación en Guipúzcoa del Colegio Oficial de Arquitectos Vasco Navarro (COAVN), ha sido financiado por el Gobierno Vasco dentro de la convocatoria ERAIKAL (https://www.euskadi.eus/y22-bopv/es/bopv2/ datos/2018/09/1804491a.shtml).

\section{REFERENCIAS}

(1) Anu Soikkelia,A. (2016). Additional floors in old apartment blocks. Energy procedia, 96: 815-823.https://doi.org/10.1016/j. egypro.2016.09.143.

(2) Instituto Nacional de Estadística, INE (2018). Nota de Prensa, Proyección de hogares 2018. Recuperado de https:// www.ine.es/prensa/ph_2018_2033.pdf

(3) Real Decreto $1053 / 1985$, de 25 de mayo, sobre ordenación de las estadísticas de las actuaciones de los Servicios contra Incendios y de Salvamento Ministerio. Boletín Oficial del Estado, núm.158, de 3 de julio de 1985, pp. 20843 a 20844. Recuperado de https://www.boe.es/diario_boe/txt.php?id=BOE-A-1985-12771

(4) Fundación MAPFRE, APTB. Víctimas de incendios en España (2010, 2011, 2012, 2013, 2014, 2015, 2016, 2017). Recuperado de https://www.fundacionmapfre.org/fundacion/es_es/publicaciones/destacadas/prevencion.jsp

(5) Fernandez-Vigil, M., y Echeverría, B. (2019). Elderly at Home: A Case for the Systematic Collection and Analysis of Fire Statistics in Spain. Fire Technology, 55, 2215-2244. https://doi.org/10.1007/s10694-019-00852-6

(6) Real Decreto 2059/1981 por el que se aprueba la Norma Básica de la Edificación «Condiciones de protección contra incendio en los edificios». Boletín Oficial del Estado núm. 224, de 18 de septiembre de 1981, pp. 21707 a 21727. Recuperado de https://www.boe.es/buscar/doc.php?id=BOE-A-1981-21124

(7) Real Decreto 279/1991 por el que se aprueba la Norma Básica de la Edificación «NBE: CPI /91: Condiciones de protección contra incendio en los edificios». Boletín Oficial del Estado núm. 58, de 8 de marzo de 1991, pp. 7911 a 7952. Recuperado de https://www.boe.es/buscar/doc.php?id=BOE-A-1991-6428

(8) Real Decreto 2177/1996 por el que se aprueba la Norma Básica de la Edificación «NBE: CPI /96: Condiciones de protección contra incendio en los edificios». Boletín Oficial del Estado núm. 261, de 29 de octubre de 1996, pp. 32378 a 32422. Recuperado de https://www.boe.es/buscar/doc.php?id=BOE-A-1996-23836

(9) Real Decreto 314/2006 por el que se aprueba el Código Técnico de la Edificación. Boletín Oficial del Estado núm. 74 de 28 de marzo de 2006, pp. 11816 a 11831. Recuperado de https://www.boe.es/buscar/doc.php?id=BOE-A-2006-5515

(10) Meacham, Brian J. (2004). Performance-Based Building Regulatory Systems: Structure, Hierarchy and Linkages. Journal of the Structural Engineering Society of New Zealand, 17(1): 37-51.

(11) Meacham, B. J., Moore, A., Bowen, R. y Traw, J. (2005). Performance-Based Building Regulation: Current Situation and Future Needs. Building Research \& Information. 33(2): 91-106. https://doi.org/10.1080/0961321042000322780

(12) Echeverría, J.B., M. Fernández-Vigil, M. y Gil, B. (2018). Dimensionado de las escaleras protegidas en caso de incendio: un reto para los métodos prescriptivos (El modelo del CTE). Informes de la Construcción, 70(550): e258. https://doi. org/10.3989/id.59396

(13) TINSCI (2018). Remuntes en edificis d'habitatges amb la creació de nous habitatges. Recuperado de http://interior. gencat.cat/web/.content/home/o3o_arees_dactuacio/bombers/prevencio_d_incendis/instruccions_guies_i_recomanacions/interpretacio_normativa_tinsci/documents/DT-14_Remuntes-i-habitatges_maig-2018-v2.pdf

(14) Ley 8/2013 de 26 de junio, de rehabilitación, regeneración y renovación urbanas. Boletín Oficial del Estado núm. 153, de 27 de junio de 2013, pp. 47964 a 48023. Recuperado de https://www.boe.es/buscar/act.php?id=BOE-A-2013-6938 
(15) MEREDICTE v1.o - Método de Evaluación del Riesgo en caso de Incendio en el Marco del Código Técnico de la Edificación. Recuperado de https://www.sttmadrid.es/archivo/normativa/normativa/incendios/meredicte-v1-o-metodo-deevaluacion-del-riesgo-en-caso-de-incendio-en-el-marco-del-codigo-tecnico-de-la-edificacion.

(16) BSI (British Standards Institution). PD 7974-6:2004 The application of fire safety engineering principles to fire safety design of buildings - Part 6: Human factors: Life safety strategies - Occupant evacuation, behavior and condition (Subsystem 6).

(17) Quintiere, J. G. (1998). Principles of Fire Behavior, Albany: Delmar Publishers.

(18) BSI (British Standards Institution). PD 7974-7:2003 Application of fire safety engineering principles to the design of buildings - Part 7: Probabilistic risk assessment.

(19) Hurley, M.J., Gottuk, D.T., Hall Jr., J.R., Harada, K., Kuligowski, E.D., Puchovsky, M., Torero, J.L., Watts Jr., J.M., Wieczorek, C.J. (Eds.) (2016). SFPE Handbook of Fire Protection Engineering. Society of Fire Protection Engineering. Nueva York: Sprinkler-Verlag. 